\title{
Rapid Clinical Promotion Model of Standardized Training for Public Health Physicians in China
}

Xiao-Yu Zhang (D)

Section of Education, Shanghai Public Health Clinical Center, Fudan University; Department of Liver Disease, Shanghai Public Health Clinical Center, Fudan University, Shanghai, 201508, People's Republic of China
Correspondence: Xiao-Yu Zhang Shanghai Public Health Clinical Center, Fudan University, Shanghai, 201508,

People's Republic of China

$\mathrm{Tel}+86$ I30 5225I333

Email zhangxiaoyuPRC@163.com
Background: Public health is an important part of the national public safety system, and the establishment of a high-quality public health team with clinical knowledge and skills is increasingly valued, especially during pandemics of infectious diseases.

Purpose: Rapid clinical promotion model of standardized training for public health physicians would be created for reference by other institutions or organizations.

Data and Methods: The data related to the standardized training of public health physicians of Grade 2019 in Shanghai were collected and sorted out, which were summarized and analyzed by interview method, field investigation method, inductive reasoning, analogy reasoning and extreme reasoning method.

Results: The rapid clinical promotion mode mainly included the following: the establishment of the management core, the definition of training objectives and implementation plans, the preparatory work in the early stage, the implementation of training missions for public health physicians, the building mechanism of tracking and feedback for clinical training, continuous follow-up and optimization of the clinical standardized training system, and the solution of key issues in clinical standardized training for public health physicians.

Conclusion: The core of the model is the vertical management of a inter-disciplinary talent to plan and implement the affairs of standardized training for public health physicians comprehensively, as well as the timely intervention of key matters.

Keywords: public health physicians, rapid clinical promotion model, standardized training

\section{Introduction}

It is well-known that public health is an important part of the national public security system. High-quality public health system is the basis for maintaining the public health, and reflects the superiority of social system and the core international competitiveness. Public health physicians are the fresh force and front sentinel of public health prevention and control. Cultivating standardized and highquality public health physicians is the foundation of the construction of public health system.

In recent years, the relationship between clinical medicine and public health has become increasingly close. ${ }^{1,2}$ Because risks and sometimes pathologies are multiple, the goal-oriented approach of medical practice encourages and assists individuals to achieve their maximal health potential in line with individually defined goals. ${ }^{3}$ And public health physicians need to be well informed about topical clinical procedures, interpersonal communication, and philosophy, as well as mastering the relevant behavioral skills. ${ }^{4}$ Therefore, this approach should thus drive public health 
doctors to shift from disease management to participatory patient management. ${ }^{5}$ In China, public health physicians are encouraged to obtain individual prescription qualifications as clinicians. ${ }^{6}$ Clinical rotation training is an important part of standardized training for public health physicians. Its core is to enable public health physicians to master clinical knowledge and skills to carry out disease prevention and control work. The goal is to train public health physicians into a standardized "medical-prevention integration and medical-prevention coordination" compound public health team.

However, until now, there has not been a model for public health physicians to participate in clinical standardized training. And public health physicians in many countries and regions around the world have not participated in or are ready to participate in clinical standardized training. How to rapidly promote the clinical training program for public health physicians and achieve the expected objects? The training experience of public health physicians of Grade 2019 in Shanghai was summarized to establish a rapid clinical promotion model of standardized training for public health physicians, so that any institutions or organizations could refer to the model to implement or further optimize the clinical training plan for cultivating standardized and high-quality public health physicians.

\section{Data and Methods}

\section{General Information}

Training data of objects of Grade 2019 who participated in the standardized training of public health physicians in Shanghai, as well as the preparatory work and management data of clinical training in the Shanghai Public Health Clinical Center. The informed consents of participants in the standardized training for public health physicians were obtained, including their data being used for the training and the research, and that this study was conducted in accordance with the Declaration of Helsinki.

\section{Definition of Standardized Training for Public Health Physicians}

Public health staff engaged in Center for Disease Prevention and Control, with a bachelor's degree or above in preventive medicine or clinical medicine, have to finish 3 months of intensive theoretical study (including 7 courses), 9 months of clinical practice (relating to the five subjects about 15 department of rotation), 12 months of public health practice (involving 19 professional work line of practice), during the training period. ${ }^{7}$ Clinical rapid promotion mode: It refers to a system that public health physicians are performed with 9 months of clinical standardized training in accordance with the outlines.

\section{Research Method}

1. Data collection: Literature, training and feedback data on the standardized training for public health physicians were collected by means of literature retrieval, internet search, questionnaire survey and field investigation.

2. Interview method: The clinical teachers, teaching secretaries of clinical departments, teaching directors of clinical departments, administrative directors of clinical departments, administrators of teaching administrative departments and management experts were interviewed on the standardized training of public health physicians at least once a week.

3. Field investigation method: On-site investigation was carried out every working day on the ward and teaching area to understand the attendance and clinical training of public health physicians. At the same time, the advice on clinical training of public health physicians and the clinical teachers are listened to and responded promptly.

4. Reasoning method: Induction reasoning method was used to classify and summarize the clinical training work and experience of standardized training for public health physicians. Analogy reasoning method was used to classify and compare the collected data to form the frame structure and specific items of the model. Extreme reasoning method was used to identify the decisive factor of every specific items of clinical rapid promotion program.

\section{Results and Notes}

The rapid clinical promotion model of standardized training for public health physicians consisted of the following seven parts. The specific framework of the model is shown in Figure 1.

\section{Establishment of the Management Core}

Appointment of full-time management personnel to take full responsibility for the training of public health physicians was a core part of the rapid promotion of the training program. 


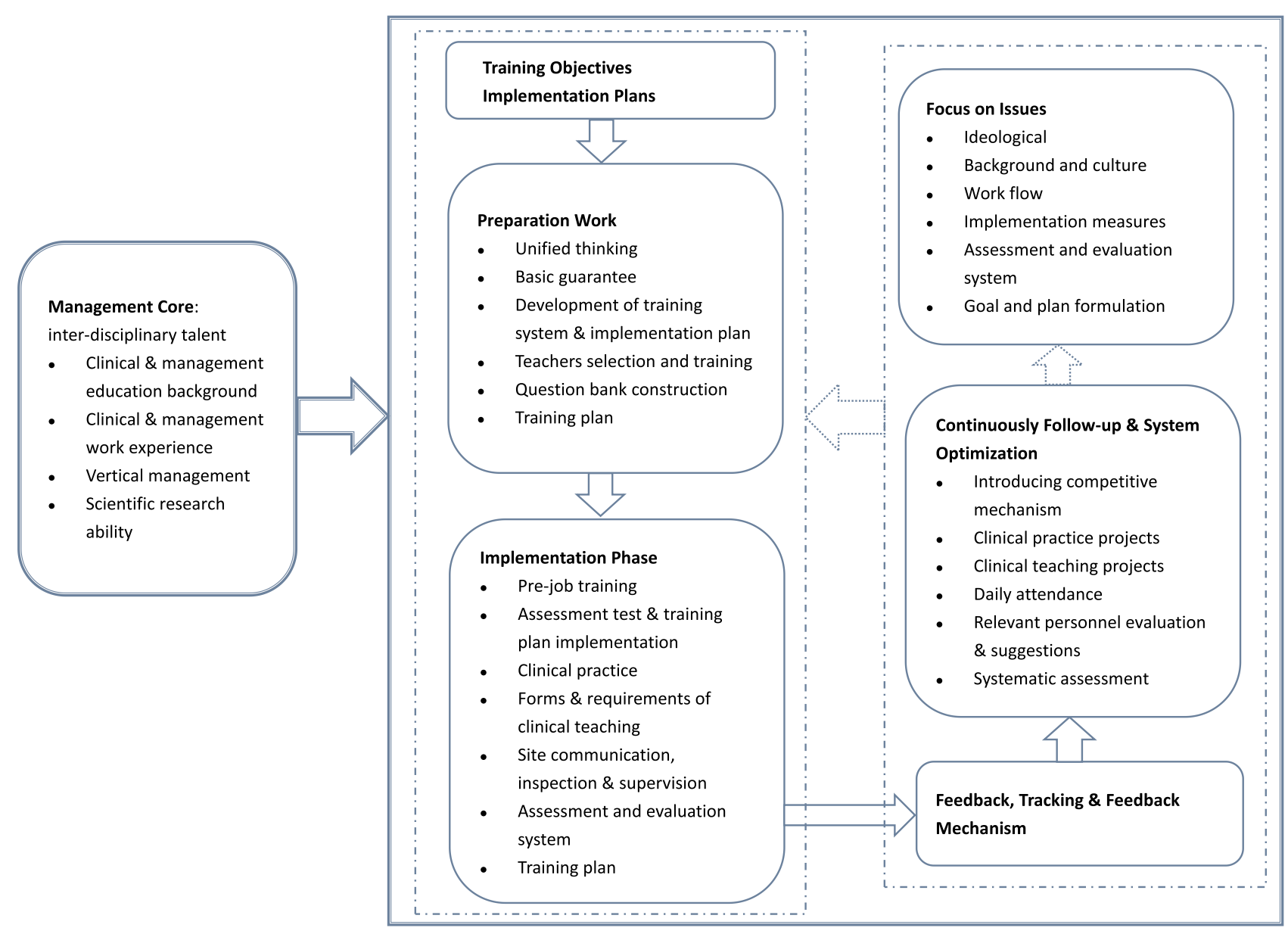

Figure I Rapid clinical promotion model of standardized training for public health physicians.

1. Full-time management personnel should not only have clinical and management education background, but also have clinical and management work experience. The double standard was to ensure that system formulation and program implementation could be quickly started and completed, and the emergency in the clinical training could be identified and intervention. All the above was also the basis for ensuring high standards and rapid promotion in clinical training.

2. During the rapid promotion of clinical training, the vertical management mechanism should be directly connected with the superior business and administrative departments, so as to make clear the training objectives, tasks and trends, and implement the relevant system formulation and program planning. In addition, it was required to enter the clinical front line and participate in training-related work.
3. Full-time management personnel should also have certain scientific research ability to ensure that clinical training affairs could be timely summarized and effectively evaluated, as well as experience sharing, during the rapid promotion of public health physician training in clinical practice.

\section{Determination of Training Objectives and Implementation Plans}

It was necessary to clarify the types of diseases, the number of cases, clinical skills and clinical thinking that public health physicians needed to master during the clinical training. In order to ensure that the standardized training work could be rapidly promoted. Relevant systems, detailed rules, indicators, question banks, training plans and assessment standards needed to be formulated, according to the training objectives and outline. 


\section{Preparation Work}

1. Unified thinking: (1) It was necessary to cultivate the important role of standardized "medicalprevention integration and medical-prevention coordination" public health physicians in public health cause, who should master basic clinical knowledge and skills. (2) It was necessary to stimulate enthusiasm for learning, and further clarify that medical literacy was the basic role of public health physicians, basic clinical knowledge and skills was a basic requirement for public health physicians to carry out their work. (3) It was necessary to increase the communication between clinicians and public health physicians, so as to learn from each other and further expand horizon of public health.

2. Basic guarantee: the status of physicians, salary guarantee, treatment subsidy, and accommodation in special areas were defined during the prescribed training period for public health physicians.

3. Development of training system and implementation plan: the standardized clinical training system and implementation plan of public health physicians would be constructed by referring to the clinical residential training experience. However, the system and plan should not be duplicated completely, they should be based on the standardized training objectives and outline of public health physicians, combined with the actual situation and requirements of the industry, and considered of the problems that may be encountered in process of rapid promotion of the project. Furthermore, the closed-loop training system, implementation plan and emergency prediction scheme would be formulated.

4. Teachers selection and training: the selection of clinical teachers for standardized training of public health physicians should be carried out, based on the actual situation and requirements of public health physicians, and avoiding high and low standards. Clinicians would be preferred as instructors with the following conditions that the clinicians had high teaching enthusiasm, rich teaching experience, high professional quality and strong teaching consciousness; and then the clinicians would be trained in batches orderly according to the plan.

5. Question bank construction: the establishment of standardized question bank was an important work for public health physicians in clinical planning and training, as a ruler for evaluating clinical training. Standardized question bank should be in accordance with the outline requirements, point to point corresponding to detailed requirements. The type of questions and difficulty degree in the question bank should be set in advance. And the question bank needed to meet the requirement of basic question quantity construction, and covered all knowledge points.

6. Training plan: In order to achieve the expected purpose of clinical training, the training tasks corresponding to the time nodes should be defined. The training rules should be implemented. And it should also be planned as a whole of department rotation, clinical practice, disease type and quantity mastery, concentrated teaching and skill operation. In addition, emergency plans should be made for special situations as well.

\section{Implementation Phase}

1. Pre-job training: (1) Routine projects: including medical core management system, hospital infection prevention and control, fire safety, spiritual civilization and organizational discipline, etc. (2) Training tasks: the objectives and rules of clinical standardized training for public health physicians should be defined, and clinical training should be carried out under the outline, and the baseline requirements should be finally completed. (3) Culture and communication: it was necessary to strengthen the publicity and education of hospital culture, department culture and clinical culture, highlighting the role positioning of public health physicians in the clinical training stage, and methods and skills of communication with clinical teaching physicians.

2. Assessment test and training plan implementation: according to the results of assessment test from the standardized question bank, the management personnel could understand the baseline profile of public health physicians about mastery of clinical knowledge, and analytical ability to diagnose and treat diseases, so as to make targeted training plans to ensure that the training plans could be carried out smoothly and achieve the expected results. The assessment test was the necessary evaluation measure during the rapid promotion of the project.

3. Clinical practice: this is a major component of clinical training for public health physicians. Firstly, 
clinical teachers should be assigned to public health physicians for clinical teaching. Secondly, hospital beds should be allocated for the public health physicians to manage inpatients as well. Thirdly, responsibilities and requirements of public health physicians for patient management were implemented. In brief, public health physicians were required to participate in the whole process of patient reception, diagnosis and treatment, operation, medical record writing and follow-up.

4. Forms and requirements of clinical teaching: in the process of clinical practice, public health physicians should attend clinical lectures, teaching rounds and case analysis according to the training plans; so that they could systematically master the clinical knowledge and application, and achieve certain professional ability to deal with clinical affairs.

5. Site communication, inspection and supervision: Management personnel needed to visit the ward and training site every weekday to check the posts of public health physicians during the rapid promotion of the program. The current training status and ideas of public health physicians could be obtained from indepth communication and exchange, and the problems encountered in the training could be solved in time to stabilize the emotions of public health physicians and good adjustments would be made to the clinical training plan. The above measures were important to ensure the smooth promotion of public health standardized training in clinical departments.

6. Assessment and evaluation system: The evaluation of public health physicians was a systematic project, which was a comprehensive evaluation of the results of the clinical training for public health physicians in clinical departments. It also provided a strong motivation for public health physicians to actively complete clinical training tasks. Especially in the rapid promotion of the project, it was necessary to pay attention to the daily attendance, the number of patients managed, the number of diseases completed and the number of operations participated by public health physicians in clinical departments. Management personnel needed to implement the assessment and evaluation work, focusing on the assessment of clinical operation, theoretical examination and case analysis, so as to urge public health physicians to achieve the clinical training objectives.

\section{Feedback, Tracking and Feedback Mechanism}

The establishment of a smooth and perfect feedback system was an important link to ensure standardized training management and training quality. Management personnel should actively communicate with public health physicians and clinical teachers, participate in clinical training inspection and supervision, and communicate with relevant personnel to evaluate the training progress and training effectiveness. From the above information to analyze the advantages and disadvantages of the training work, in order to timely handling with the problems occurred in the clinical training process and achieve the objectives. The whole operation mechanism and the reserve information were of great value to the optimization and construction of the follow-up plan and training system.

\section{Continuously Follow-Up and System Optimization}

Continuous follow-up and system optimization of clinical training for public health physicians was an important part of the standardized training. However, in the stage of rapid promotion, these projects were preset plans, which were in the process of information storage and needed to be completed after evaluation. The follow-up of feedback information was to prepare for further improvement in the later stage. The task of this stage was mainly to provide experience accumulation, which should be set up a model for rapid promotion and set as a follow-up optimization project.

Key points of follow-up and optimization: (1) Introducing competitive mechanism: assessment and grading of public health physicians should be given, setting up excellent, qualified, unqualified, or setting the pass rate, which could stimulate the learning motivation and competitive awareness of public health physicians; while reward and punishment system should be set up for clinical teacher to stimulate their enthusiasm in teaching. (2) Clinical practice projects: relevant measures were further optimized according to the completion status, quality and results of the clinical training tasks of public health physicians in the early stage. (3) Clinical teaching projects: appropriate improvement could be made according to the teaching and training effect and the feedback of public health physicians, so as to achieve the smooth progress of clinical training. (4) Daily attendance: in the rapid promotion stage of the project, daily attendance was still the focus of process management. (5) Relevant personnel 
evaluation and suggestions: early communication and suggestions could be finished on clinical practice, teaching plan and working process through face-to-face or communication tools, and problems should be solved in the bud, which was also an important reference for subsequent system and process improvement. (6) Systematic assessment: it mainly included process evaluation and clinical department evaluation. Process assessment was an important part of standardized training management. It was the prerequisite to determine whether public health physicians could participate in the after-department examination, which ensured the timely completion of the planned objectives during the clinical standardized training. In the rapid promotion stage, the clinical department assessment mainly consisted of theoretical examination, case analysis and clinical operation. All assessment criteria should have quantifiable indicators.

\section{Focus on Issues}

In the rapid promotion stage of standardized training for public health physicians, training work should be carried out according to the training content and requirements of the standardized training outline for public health physicians, which should not be changed by any organization or individual.

1. Ideological aspects: The orientation of clinical standardized training stage for public health physicians was the training object. Clinical training was established on the basis of policy, and it was not changed by personal consciousness. The purpose of clinical training was to learn and master clinical knowledge and skills. The spirit of being willing to be ascetic was required in the training process, in order to obtain the maximum clinical knowledge reserve in the limited clinical training time. It was necessary to propagandize and guide in the pre-job training and process management, take the examination as the interest orientation, and finally achieve the clinical training effect as the goal.

2. Background and culture: Public health physicians mainly focused on public health prevention and control and lacked of scheme and experience in diagnosis and treatment of diseases, which made it difficult for them to have a sense of clinical belonging. Therefore, it was important for public health physicians to pay attention to and be familiar with the clinical routine diagnosis and treatment mode, experience and clinical culture, which was of great significance for public health physicians to achieve the smooth progress of clinical training work.

3. Work flow: Working environment of public health physicians was very different from that of clinical training places, and each clinical department had its own characteristics. Public health physicians must have a positive learning attitude, follow the work pace of the clinical teacher closely, and pay attention to each clinical dynamic and treatment methods, In order to obtain the maximum clinical knowledge and skills from clinical standardized training.

4. Implementation measures: In the process of rapid promotion, it was necessary to grasp the main problems, not covering all aspects. According to the training plan, the rhythm of clinical standardized training should be well controlled and the key indexes should be grasped. Key indicators of clinical training should be completed according to time nodes, and administrative intervention was required in case of emergency. In the case of the completion of key tasks, appropriate attention could be paid to the progress and feasibility of relevant indicators, and it was not necessary to list all items that would be completed in the rapid promotion stage.

5. Goal and plan formulation: In the rapid promotion stage of the project, we must be rigorous and pragmatic, not to bring those impractical or irrelevant projects into clinical training. According to the actual situation of the public health physicians and the actual clinical training ability, clinical training should avoid the blind pursuit of advanced items, sophisticated items, hot items, scientific research items and management items; which would deviate from the original intention of clinical professional training, and affect the overall clinical training time and training effect.

\section{Discussion}

The purpose of clinical training is to further consolidate clinical knowledge and acquire the ability to deal with clinical affairs through the clinical standardized training for public health physicians. Because public health physicians are very different from clinicians in terms of specialty and working environment, it is necessary to carry out personalized and systematic planning for promotion of their clinical training. This paper systematically summed up the experience of clinical training for public health physicians from preparation 
work, training program, the detailed rules for the implementation and special training work. And a variety of analysis methods were applied to summarize the clinical training experience to establish rapid clinical promotion model of standardized training for public health physicians, for other institutions or organizations that have not yet implemented or will be ready to implement clinical training for public health physicians to provide the reference and guidance.

\section{Important Components and Key Points of the Model}

The composition and operation of the rapid clinical promotion model of standardized training for public health physicians includes: establishing a management core for clinical standardized training, formulating training objectives and implementation plans, making comprehensive preparations for the clinical training, carrying out clinical training for public health physicians according to the objectives and plans, setting up a feedback mechanism for the whole clinical training process, constantly following up the progress and optimizing the training system of clinical standardized training, and solving key issues in clinical standardized training for public health physicians. The key points of the model is that a inter-disciplinary talent is appointed for vertical management to organize and implement the affairs of clinical standardized training for public health physicians comprehensively, as well as the timely intervention of key matters.

\section{Comparison with Existing Literature}

At present, some countries represented by the United States had achieved relatively mature standardized training for public health physicians in the world, which was generally characterized by long training time and high standard requirements. Medical education in the United States was positioned as elite education, and undergraduate graduation was determined as the admission requirement. ${ }^{8}$ In the United States, one must first obtained a medical degree and a medical practitioner's license to become a preventive medicine physician. Combined with the time required for residency training and preventive medicine specialist training, it took at least 13 years to become a preventive medicine physician. ${ }^{9-11}$ From the perspective of the quality of education and training, American preventive medicine physicians possessed basic clinical diagnosis and treatment skills, and to some extent they were at least qualified doctors who could cure diseases and save lives. ${ }^{12}$
In the clinical rapid promotion mode which was established in this paper, through the formulation of training rules and implementation plan, public health physicians could acquire basic clinical management ability after 9 months of standardized clinical training. So the model had better repeatability and would be easier to be popularized.

Another study showed that preventive medicine education in some countries could be regarded as linear sum of clinical medical education and public health education, but at the expense of sacrificing students' mastery of some clinical skills for mastery of public health skills in actual medical education; in a certain historical period, it could quickly to cultivate a large number of public health personnel directly involved in the health and epidemic prevention. $^{13}$ The rapid clinical promotion mode could enable public health physicians to acquire a certain level of clinical business handling ability after 9 months of standardized clinical training, and that comprehensively improved the core competence and practical application ability of public health physicians.

\section{Strengths of the Model}

The clinical training period of this model is relatively short, only 9 months in total; while the existing mature model of clinical training cycle is too long in other countries, such as USA. Public health physicians could obtain basic clinical transaction management ability after 9 months of standardized clinical training from the model in China. The research data was obtained from the management department, clinical front-line work, and expert interviews, and various induction, analogy and extreme reasoning methods were used to analyze and summarize the research data. Such model had high practicality and replicability. This model focuses on the preparatory work, implementation plan, working process and key concerns of the planning and training; and this paper also gave a detailed explanation of the model, and offered a supplement to some problems that were easy to be ignored and improper during the clinical training of public health physicians; all the above of this model would be very helpful to be applied in other countries or regions.

\section{Limitations of the Model}

The purpose of this model was to provide a standardized system for the rapid promotion of clinical training for public health physicians, but there were also some aspects that needed to be further supplemented and perfected. Under this mode, the 
electronic systems were not applied to the standardized training of public health physicians. In the later stage, electronic platform would be introduced to conduct the standardized training and management of public health physicians, so as to improve the management and operation efficiency. Moreover, the model did not include the prescribing rights of doctors' qualifications in the standardized training of public health physicians; and in the later stage, doctors' prescribing qualifications would be gradually connected with the standardized training of public health physicians.

\section{Conclusion}

The core of the model is that a inter-disciplinary talent is appointed for vertical management to plan and implement the affairs of standardized training for public health physicians comprehensively, as well as the timely intervention of key matters.

\section{Literature Share Statement}

The model included in the manuscript submitted to the journal is transparent. Consent from the corresponding author is required for any institution or individual to reprint this document.

\section{Ethical Approval and Consent to Participate}

Informed consents of participants in the standardized training for public health physicians were obtained for the training and the study. All the data received Institutional Review Board (IRB) approval by the Ethics Committee. The IRB number was No. 2021-S026-01.

\section{Consent for Publication}

The author has read and agreed to the published version of the manuscript.

\section{Acknowledgments}

This study was supported by the standardized training teams for public health physicians.

\section{Author Contributions}

The author made substantial contributions to conception and design, acquisition of data, or analysis and interpretation of data; took part in drafting the article or revising it critically for important intellectual content; agreed to submit to the current journal; gave final approval of the version to be published; and agree to be accountable for all aspects of the work.

\section{Funding}

This research received no external funding.

\section{Disclosure}

The author declares no conflicts of interest.

\section{References}

1. Frieden TR. SHATTUCK LECTURE: the future of public health. $N$ Engl J Med. 2015;373(18):1748-1754. doi:10.1056/NEJMsa1511248

2. Rao R, Hawkins M, Ulrich T, Gatlin G, Mabry G, Mishra C. The evolving role of public health in medical education. Front Public Health. 2020;8:251. doi:10.3389/fpubh.2020.00251

3. Mold JW, Blake GH, Becker LA. Goal-oriented medical care. Fam Med. 1991;23(1):46-51.

4. Unger JP, Morales I, De Paepe P, Roland M. Integrating clinical and public health knowledge in support of joint medical practice. $B M C$ Health Serv Res. 2020;20(Suppl2):1073. doi:10.1186/s12913-02005886-z

5. De Maeseneer J, Boeckxstaens P. James Mackenzie Lecture 2011: multimorbidity, goal-oriented care, and equity. $\mathrm{Br} J$ Gen Pract. 2012;62(600):e522-e524. doi:10.3399/bjgp12X652553

6. Lei J, Luo HM, Dai Z, Ma J. Reflections on the orientation of public health physicians. Zhonghua Yu Fang Yi Xue Za Zhi. 2020;54 (1):13-17. doi:10.3760/cma.j.issn.0253-9624.2020.01.005

7. Lu H, He Q, Chen Y, Sun R, Pan Q, Yuan D. Exploration on the core elements of public health medicine standardized training program in institutes of disease control and prevention in China. Chinese Health Res. 2020;23(1):68-71,77. doi:10.13688/j.cnki.chr.2020.19576

8. Duffy TP. The Flexner Report-100 years later. Yale J Biol Med. 2011;84(3):269-276.

9. Liaison Committee on Medical Education. Medical school directory [EB/OL]. Available from: https://lcme.org/directory/. Accessed February 14, 2020

10. United States Medical Licensing Examination. Bulletin of information[EB/OL]. Available from:. https://www.usmle.org/bulletin/. Accessed December 14, 2019.

11. Accreditation Council for Graduate Education. Accreditation Council for Graduate Medical Education (ACGME)-public[EB/OL]. Available from: https://apps.acgme.org/ads/Public. Accessed November 28, 2019.

12. Hilliard TM, Boulton ML. Public health workforce research in review: a 25-year retrospective. Am J Prev Med. 2012;42(5 Suppl 1):S17-S28. doi:10.1016/j.amepre.2012.01.031

13. Li G, Luo W-J, Feng Z-C. Policy evolution analysis on construction of Chinese public health physician system. Chinese Health Resources. 2019;22(3):242-245. doi:10.3969/j.issn.1007-953X.2019. 03.015 


\section{Publish your work in this journal}

Advances in Medical Education and Practice is an international, peerreviewed, open access journal that aims to present and publish research on Medical Education covering medical, dental, nursing and allied health care professional education. The journal covers undergraduate education, postgraduate training and continuing medical education including emerging trends and innovative models linking education, research, and health care services. The manuscript management system is completely online and includes a very quick and fair peer-review system. Visit http://www.dovepress.com/testimonials.php to read real quotes from published authors.

Submit your manuscript here: http://www.dovepress.com/advances-in-medical-education-and-practice-journal 